\title{
Clinical usefulness and feasibility of time-frequency analysis of chemosensory event-related potentials*
}

\author{
C. Huart ${ }^{1,2}$, Ph. Rombaux ${ }^{1,2}$, T. Hummel ${ }^{3}$, A. Mouraux ${ }^{2}$ \\ ' Department of Otorhinolaryngology, Cliniques universitaires Saint-Luc, Brussels, Belgium \\ ${ }^{2}$ Institute of Neuroscience, Université catholique de Louvain, Brussels, Belgium \\ ${ }^{3}$ Smell \& Taste Clinic, Technical University Dresden Medical School, Dresden, Germany
}

\author{
Rhinology 51: 210-221, 2013 \\ DOI:10.4193/Rhino13.007 \\ *Received for publication: \\ January 22, 2013
}

Accepted: April 22, 2013

\begin{abstract}
Summary
Background: The clinical usefulness of olfactory event-related brain potentials (OERPs) to assess olfactory function is limited by the relatively low signal-to-noise ratio of the responses identified using conventional time-domain averaging. Recently, it was shown that time-frequency analysis of the obtained EEG signals can markedly improve the signal-to-noise ratio of OERPs in healthy controls, because it enhances both phase-locked and non phase-locked EEG responses. The aim of the present study was to investigate the clinical usefulness of this approach and evaluate its feasibility in a clinical setting.
\end{abstract}

Methodology: We retrospectively analysed EEG recordings obtained from 45 patients ( 15 anosmic, 15 hyposmic and 15 normos$\mathrm{mic}$ ). The responses to olfactory stimulation were analysed using conventional time-domain analysis and joint time-frequency analysis. The ability of the two methods to discriminate between anosmic, hyposmic and normosmic patients was assessed using a Receiver Operating Characteristic analysis.

Results: The discrimination performance of OERPs identified using conventional time-domain averaging was poor. In contrast, the discrimination performance of the EEG response identified in the time-frequency domain was relatively high. Furthermore, we found a significant correlation between the magnitude of this response and the psychophysical olfactory score.

Conclusion:Time-frequency analysis of the EEG responses to olfactory stimulation could be used as an effective and reliable diagnostic tool for the objective clinical evaluation of olfactory function in patients.

Key words: chemosensory event-related potentials, olfaction, trigeminal, time-frequency analysis, EEG

\section{Introduction}

Olfaction has received less attention from researchers than our other senses. This is partly due to the technical challenge of working with odorous stimuli and the difficulties of measuring brain activity induced by a chemosensory stimulus. Nevertheless, the sense of smell plays a major role in the detection of potential environmental threats, nutrition, social behaviour, well-being and also memory processes ${ }^{(1)}$.

Recent studies have shown that olfactory disorders are extre- mely common, affecting up to $20 \%$ of the general population (2). Although olfactory dysfunction is often unnoticed by the patient, it has been shown to impact significantly on quality of life ${ }^{(3)}$. It is widely accepted that the evaluation of patients with olfactory dysfunction requires a precise clinical work-up to 1) assess olfactory function, 2) determine the aetiology of olfactory dysfunction and 3) provide adequate treatment, prognosis and counselling to patients.

The evaluation of olfactory function is most often performed 
using psychophysical testing such as the Sniffin' Sticks test ${ }^{(4,5)}$ or the University of Pennsylvania Smell Identification Test (UPSIT) (6). These tests have the advantage of being easy to implement, of being validated in multicenter studies ${ }^{(4,7)}$ and of having a high test-retest reliability ${ }^{\left({ }^{8}\right)}$. However, these tests have the disadvantage of being subject to potential patient response bias. This constitutes - at least in some situations - a major drawback when evaluating patients complaining of an olfactory disorder. The recording and assessment of chemosensory event-related potentials (CSERPs) has therefore been proposed as an alternative method to evaluate nasal chemosensory function. As compared to psychophysical testing, electrophysiological testing only requires some collaboration by the subject/patient. This is of particular interest, for example, in patients with cognitive dysfunction, children or assessments performed in a medicolegal context ${ }^{(9-11)}$.

Until now, CSERPs have been mainly investigated using conventional time-domain averaging ${ }^{(12-14)}$. Unfortunately, olfactory event-related potentials (OERPs) exhibit a low signal-to-noise ratio ${ }^{(15-17)}$ and, probably for this reason, cannot be reliably identified in approximately $30 \%$ of normosmic subjects ${ }^{(15)}$. Hence, the clinical usefulness of OERPs remains, at present, limited.

Recently, we have shown that a time-frequency analysis of the recorded electroencephalographic signals (EEG) can markedly improve the signal-to-noise ratio of CSERPs, in particular, OERPs recorded in healthy controls ${ }^{(18)}$. The technique, based on the continuous wavelet transform, has the advantage of revealing EEG responses that are not strictly phase-locked to the stimulus onset. Hence, the approach is 1) less sensitive to temporal jitter and 2) allows characterising non-phase locked EEG responses such as event-related synchronisation (ERS) and desynchronisation (ERD). Therefore, it could allow identifying EEG responses which cannot be consistently identified using conventional time-domain averaging. Indeed, the low signal-to-noise ratio of OERPs could at least in part be due to temporal jitter, resulting from the relatively long latency between the onset of the olfactory stimulus and the generation of a cortical response. Furthermore, we found that, in healthy subjects, the magnitude of the EEG responses identified in the time-frequency domain correlated significantly with the psychophysical olfactory performance assessed using the Sniffin' Sticks test.

Here, our aim was to 1) assess the clinical usefulness of the time-frequency analysis of EEG responses to chemosensory stimulation and 2) evaluate the feasibility of implementing this technique in a clinical setting.

\section{Materials and methods}

Participants
We retrospectively analysed EEG recordings obtained from 45 patients ( 20 females, 25 males), aged between 18 and 64 years, that presented themselves or were referred to the smell dysfunction clinic of our department. All patients had subjective complaints of olfactory disorder. Based on a psychophysical evaluation, 15 patients were considered as normosmic (8 females and 7 males aged $15-62$ years; $41 \pm 14$; mean \pm SD), 15 patients were considered as hyposmic ( 7 females and 8 males aged 21-61 years; $42 \pm 13$ ) and 15 patients were considered as functionally anosmic (in the following addressed simply as "anosmic") (6 females and 9 males aged 18-64 years; $42 \pm 14$ ). The three groups were similar in terms of age (one-way ANOVA; $p=0.899$ ) and sex (chi-square test; $\mathrm{p}=0.765$ ).

Normosmic patients presented to the smell clinic for various reasons: suspected post-infectious olfactory disorder (8 patients), suspected post-traumatic olfactory disorder (2 patients), glossodynia (2 patients), hyperacousia (1 patient), hypogonadism in order to exclude a Kallmann's syndrome (1 patient), family history of congenital anosmia (1 patient) and dry syndrome (1 patient). Hyposmic and anosmic patients suffered from post-infectious olfactory loss ( 9 and 5 patients) or post-traumatic olfactory loss (6 and 10 patients).

The investigations were approved by the local Ethics committee.

\section{Psychophysical evaluation}

Psychophysical orthonasal olfactory function was assessed using the validated Sniffin' Sticks test ${ }^{(4,5)}$. In this test, odours are presented to the subjects using felt-tip pens placed approximately $2 \mathrm{~cm}$ in front of both nostrils, as follows. First, the olfactory threshold $(T)$ is assessed using $n$-butanol presented by means of a single staircase procedure, using stepwise dilutions in a row of 16 felt tip pens. Second, odour discrimination (D) is assessed by asking subjects to perform a 3-alternative forced choice (3-AFC) task using 16 pairs of odorant. Third, odour identification (I) is assessed by asking the subject to identify 16 individual odours by performing a forced choice from a list of four verbal descriptors. Olfactory threshold (T), discrimination (D) and identification (I) are then added together to give the TDI score ${ }^{(4,5)}$, used to define normosmia (TDI $\geq 31)$, hyposmia $(16 \leq \mathrm{TDI}<31)$ and anosmia $(\mathrm{TDI}<16)^{(19)}$.

The mean TDI score was 34.5 (95\% confidence interval: 32.936.1 ), ranging from 31 to 40 in the normosmic group, 23.0 (95\% confidence interval: 20.6-25.4), ranging from 16 to 30 in the hyposmic group and 11.1 (95\% confidence interval: 9.3-12.8), ranging from 5 to 15 in the anosmic group.

\section{Stimuli}

Chemosensory stimuli were produced by an air-dilution olfactometer (OM2S, Burghart Medical Technology, Wedel, Germany). 
The device is able to deliver brief pulses of odorants embedded in a constant airflow. The rapid switching between the odorant airflow and the control airflow is based on a vacuum line. During the stimulation, the airflow $(8 \mathrm{l} / \mathrm{min})$, temperature $\left(36^{\circ} \mathrm{C}\right)$, and humidity ( $80 \%$ relative humidity) remain constant. This avoids any concomitant stimulation of mechanical or heat sensitive trigeminal receptors. Olfactory stimuli were generated by 2-Phenylethanol $(50 \% \mathrm{v} / \mathrm{v})$, and trigeminal stimuli were generated by gaseous $\mathrm{CO} 2(55 \% \mathrm{v} / \mathrm{v})^{(20,21)}$. These two types of stimuli activate selectively olfactory and trigeminal afferents, respectively. The stimuli were delivered through a TeflonTM tube placed in the right nostril, with its ending just behind the nasal valve, pointing towards the olfactory cleft. Stimulus duration was $200 \mathrm{~ms}$ with a rise time of $20 \mathrm{~ms}$.

\section{Procedure}

Before the experiment, subjects were familiarized with the experimental surrounding, the material used for the psychophysical assessment of olfaction, as well as the olfactory and trigeminal stimuli used to elicit chemosensory ERPs. During the experiment, olfactory and trigeminal stimuli were presented in alternation. Each type of stimulus was repeated 20 times. Interstimulus interval between each stimulus was $30 \mathrm{~s}$. Hence, the time interval between two stimuli of the same type was $60 \mathrm{~s}$. Subjects were instructed to breathe through the mouth and to perform velo-pharyngeal closure to avoid any respiratory airflow in the nasal cavity during stimulus presentation ${ }^{(22)}$. Subjects sat in a comfortable chair located in a well-ventilated room. They were instructed to keep their eyes open during the recording.

\section{EEG recording}

The EEG was recorded continuously at a $256 \mathrm{~Hz}$ sampling rate from scalp electrodes located the International 10-20 positions Fz, Cz, Pz. The signals were amplified and digitised using a SAM 32EP system (Micromed, Mogliano Veneto, Italy). Linked earlobes (A1/A2) were used as reference. Ocular movements and eye-blinks were recorded using two additional bipolar surface electrodes placed at the upper-left and lower-right side of the left eye. The impedance of all electrodes was always kept below $10 \mathrm{kOhm}$.

\section{Data preprocessing}

All EEG processing steps were carried out using Letswave 4 (http://nocions.webmode.com/letswave) ${ }^{(23)}$. After band-pass filtering using a 0.3 to $30 \mathrm{~Hz}$ Butterworth zero phase filter, the continuous EEG recordings were segmented into 2.0 s long EEG epochs ranging from -0.5 to +1.5 s relative to stimulus onset. After baseline correction (reference interval: -0.5 to $0 \mathrm{~s}$ ), epochs with amplitude value exceeding $\pm 50 \mu \mathrm{V}$ were rejected. A minimum of $50 \%$ artefact-free EEG epochs (10 trials) was considered as the limit allowing further interpretation of the elicited EEG responses. Twelve subjects (four in each group) were rejected for this reason.

\section{Across-trial averaging in the time domain}

For each subject, separate average waveforms were computed for olfactory and trigeminal stimulation. Within all average waveforms (including the waveforms in which no peaks could be clearly identified visually), two distinct peaks were measured at electrode $\mathrm{Cz}$ using the following objective criteria. For olfactory CSERPs, the $\mathrm{N} 1$ was measured as the most negative amplitude value between 320 and 450 ms (OLF-N1) and the P2 was defined as the most positive amplitude value between 450 and $800 \mathrm{~ms}$ (OLF-P2) $^{(12,20,21,24-26)}$. For trigeminal CSERPs, the N1 was measured as the most negative amplitude value between 320 and $450 \mathrm{~ms}$ (TRI-N1) and the P2 was defined as the most positive amplitude value between 450 and $800 \mathrm{~ms}$ (TRI-P2)(12,20,24,25). Latencies were expressed relative to stimulus onset. Amplitudes were expressed relative to baseline.

Across-trial averaging in the time-frequency domain Similarly to our previous study, a time-frequency (TF) representation based on the continuous Morlet wavelet transform (CWT) of EEG epochs was used to characterise the amplitude of oscillatory activity as a function of time and frequency ${ }^{(18)}$. The Morlet wavelet consists in a complex exponential function localised in time by a Gaussian envelope. The initial spread of the Gaussian wavelet was set at $2.5 / \pi \omega 0$ ( $\omega 0$ being the central frequency of the wavelet (see also ${ }^{(23,27)}$ ). Explored frequencies ranged from 0.3 to $30 \mathrm{~Hz}$ in steps of $0.3 \mathrm{~Hz}$.

To obtain a time-frequency representation of trigeminal and olfactory CSERPs, the time-frequency transform was first applied to the single-subject ERP waveforms obtained after time-domain averaging (CWT-AVERAGE). Because time-domain averaging cancels out signal changes that are not strictly stationary across trials, this transform revealed only stimulus induced EEG changes that were phase-locked to the stimulus onset (i.e. ERPs).

To obtain a time-frequency representation of both phase-locked and non phase-locked EEG responses to trigeminal and olfactory stimulation, the time-frequency transform was then applied to each single EEG epoch (CWT-SINGLE). For each subject and stimulus type, single-trial TF maps expressing signal amplitude were then averaged across trials. Because this approach yields a time-frequency map of the average oscillation amplitude regardless of the phase, it enhanced both phase-locked (i.e. ERPs) and non phase-locked (i.e. ERD, ERS, ERPs affected by a significant amount of latency jitter) stimulus-induced changes in EEG oscillation amplitude.

For each estimated frequency, CWT-AVERAGE and CWT-SINGLE 
time-frequency maps were expressed relative to baseline (prestimulus interval ranging from -0.4 to $-0.1 \mathrm{~s}$ relative to stimulus onset), as follows: ER\%tf = (At,f-Rf)/Rf, where At, $f$ is the signal amplitude at a given latency $t$ and frequency $f$, and $R f$ is the signal amplitude at the frequency $f$, averaged within the prestimulus reference interval.

Based on our previous findings, we defined time-frequency regions of interest (ROIs) in the CWT-SINGLE time-frequency maps wherein we measured the magnitude of the EEG responses to chemosensory stimulation. The ROls corresponded to the ROls that were associated with the highest discrimination performance in our previous study performed in healthy participants(18). That is, 300-1000 ms and 3-7 Hz for olfactory EEG responses (measured at Fz) and 200-600 ms and 2-7.5 Hz for trigeminal EEG responses (measured at $\mathrm{Cz}$ ).

\section{Statistical analysis}

\section{Group comparisons}

Group-level statistical analyses were performed using SPSS

17.0 (SPSS Inc, Chicago, IL, USA). A one-way ANOVA was used to compare the measures obtained in each of the three different groups (anosmic, hyposmic, normosmic). When significant, pairwise post-hoc t-tests were performed using a Bonferonni correction for multiple comparisons. The level of significance was set at $\mathrm{p}<0.05$.

\section{Discrimination performance}

The aim of the present study was to evaluate the clinical usefulness of a time-frequency analysis of the EEG responses to chemosensory stimulation in a population of patients presenting with olfactory complaints. For this purpose, we constructed Receiver Operating Characteristic (ROC) curves to examine and compare the ability of each of the different measures of the EEG responses to olfactory stimulation to discriminate between three different groups of patients, anosmic, hyposmic and normosmic patients, defined based on their TDI scores. Specifically, we examined the ability to discriminate between: 1) normosmic and hyposmic/anosmic patients; 2 ) normosmic and hyposmic patients; and 3) normosmic and anosmic patients. These analyses were performed using MedCalc v. 11.5 (MedCalc Software, Mariakerke, Belgium). The area under the ROC curve (AUC) was used as an index of discrimination performance. An AUC of 0.5 indicates random performance, whereas an AUC of 1 and 0 denotes perfect performance. For each measure, the ability to distinguish between different groups of patients, was assessed by examining whether the AUC was significantly different from $0.5^{(28,29)}$. When significant $(p<0.05)$, the cutoff value $(J)$ associated with the greatest Youden index $(y=$
Sensitivity+Specificity-1) was chosen as decision criterion ${ }^{(30,31)}$. This cutoff value corresponds to the point on the ROC curve that is the farthest from the diagonal line. Finally, to compare the discrimination performance of the different EEG measures, the ROC curves were compared using a nonparametric approach for comparing areas under two or more correlated ROC curves, as described by Delong et al. ${ }^{(32)}$.

\section{Correlation}

To examine the relationship between the different measures of the EEG response to olfactory and trigeminal stimulation and chemosensory perception, the correlation between the magnitude of the elicited EEG responses and the TDI score was assessed using Spearman's rank correlation coefficient. These analyses were performed using SPSS 17.0 (SPSS Inc, Chicago, III., USA).
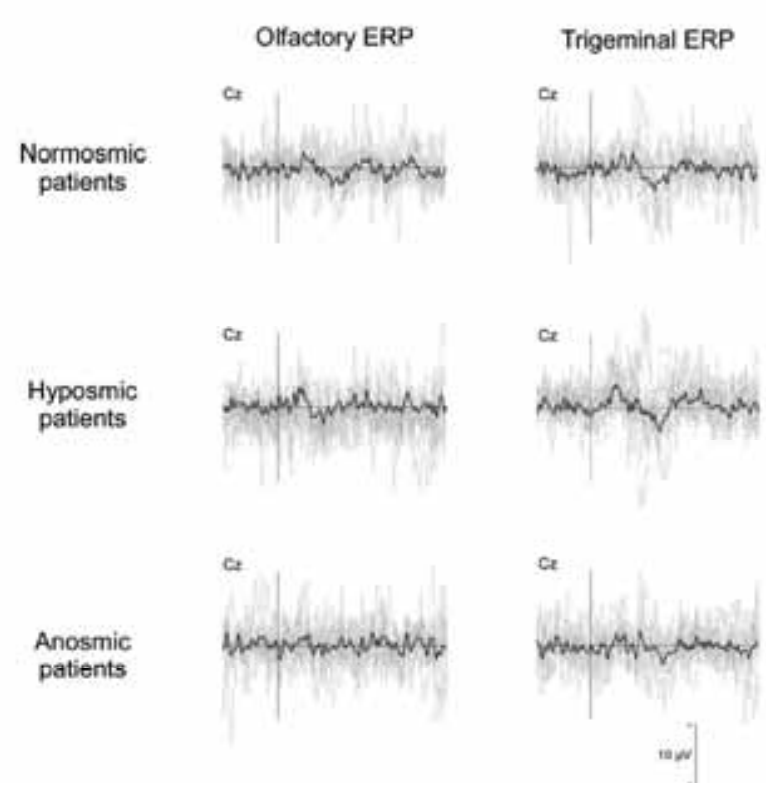

Figure 1. Olfactory and trigeminal ERPs. Olfactory and trigeminal ERPs recorded at the scalp vertex ( $C z$ vs $A 1 A 2)$ in 11 normosmic, 11 hyposmic and 11 anosmic patients. 2-phenylethanol (50\% v/v) was used to selectively activate olfactory afferents. Gaseous $\mathrm{CO} 2(50 \% \mathrm{v} / \mathrm{v})$ was used to selectively activate trigeminal afferents. 20 stimuli were presented, lasting 200ms (20-ms rise time), separated by a 60 s inter-stimulus interval. Individual ERP waveforms are shown in light grey. The group-level average waveform is shown in black. 
Olfactory stimulation
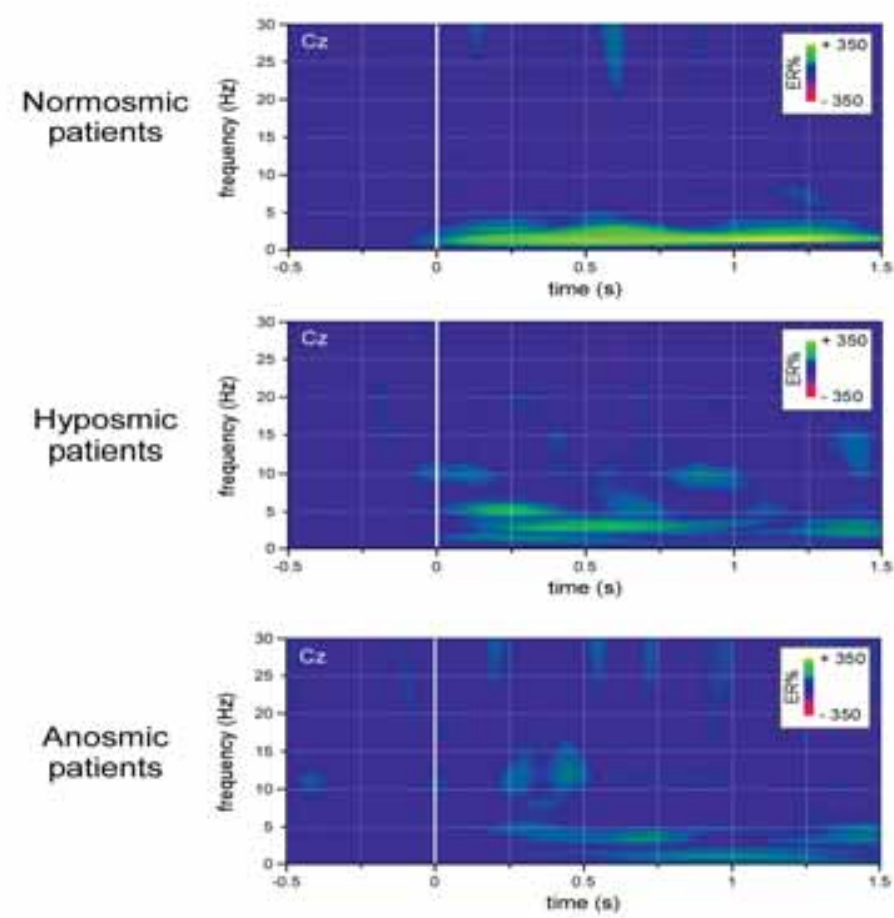

Trigeminal chemosensory stimulation
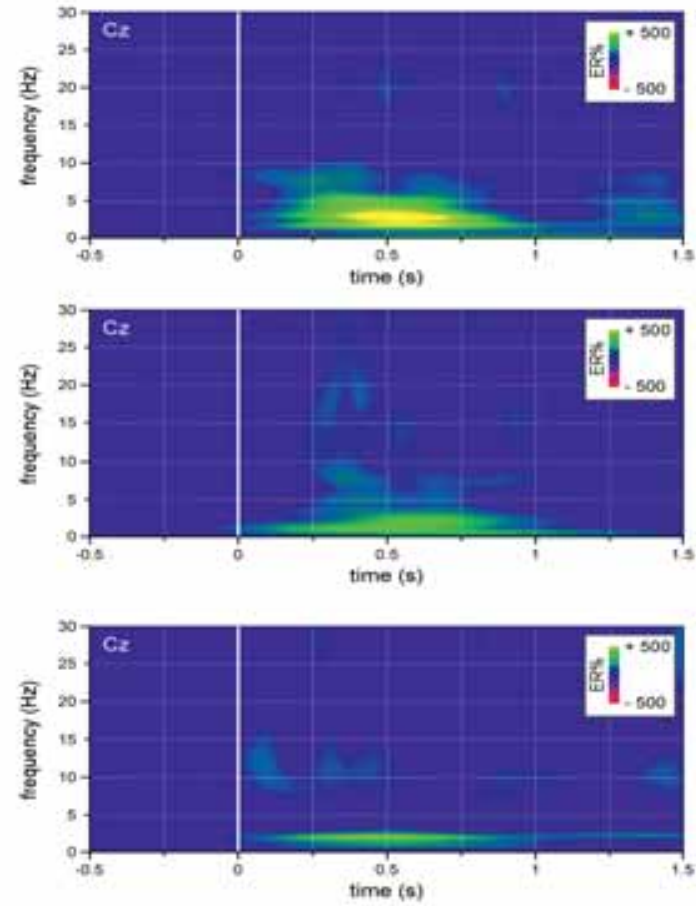

Figure 2. Time-frequency representation of the phase-locked EEG responses to olfactory and trigeminal stimulation (CWT-AVERAGE) in 11 normosmic, 11 hyposmic and 11 anosmic patients. The time-frequency transform of the waveforms obtained by performing conventional across trial averaging in the time-domain was used to identify phase-locked EEG responses. Signal amplitude (group-level average, electrode Cz vs. A1A2) is expressed as percentage increase or decrease relative to baseline (-0.4 to -0.1s) (ER\%). In normosmic patients, the olfactory ERP is represented as an increase of low-frequencies activities. This phase-locked activity is less pronounced in hyposmic patients and hardly identifiable in anosmic patients. In contrast, the trigeminal ERP is clearly identifiable in all three groups of patients. Its amplitude appears reduced in the anosmic group. However, this reduction was not significant.

\section{Results}

Olfactory stimulation

\section{Across trial averaging in time domain}

Olfactory stimulation elicited, at electrode $\mathrm{Cz}$, a small negative deflection (OLF-N1) followed by a positive deflection (OLF-P2) (Figure 1) which was visually-identifiable in 6 out of the 11 normosmic patients, 4 out of the 11 hyposmic patients and only 1 out of the 11 anosmic patients.

The latency of OLF-N1 was $357 \pm 32,398 \pm 42$ and $383 \pm 38$ ms in the normosmic, hyposmic and anosmic waveforms, respectively. The latency of OLF-P2 was $566 \pm 101,570 \pm 102$ and 678 $\pm 90 \mathrm{~ms}$ in the normosmic, hyposmic and anosmic waveforms, respectively.

The amplitude of OLF-N1 was $-3.4 \pm 2.2,-2.7 \pm 2.0$ and $-3.3 \pm$ $2.3 \mu \mathrm{V}$ in the normosmic, hyposmic and anosmic waveforms, respectively. The amplitude of OLF-P2 was $4.9 \pm 2.1,4.0 \pm 1.6$ and $5.2 \pm 2.2 \mu \mathrm{V}$ in the normosmic, hyposmic and anosmic waveforms, respectively (Table 1). These differences were not significant (OLF-N1: $F=0.350 ; p=0.707 ;$ OLF-P2: $F=1.033 ; p=$
0.368).

Neither the amplitude of OLF-N1 nor the amplitude of OLF-P2 (estimated in each single-subject ERP waveform) were able to discriminate 1) between normosmic and hyposmic/anosmic patients (OLF-N1: AUC $=0.56 \pm 0.11, p=0.600$; OLF-P2: AUC = $0.54 \pm 0.11, p=0.668) ; 2$ ) between normosmic and hyposmic patients (OLF-N1: AUC $=0.59 \pm 0.13, p=0.461$; OLF-P2: AUC $=$ $0.62 \pm 0.13, p=0.344)$; and 3 ) between normosmic and anosmic patients (OLF-N1: AUC $=0.52 \pm 0.13, p=0.874$; OLF-P2: $A U C=$ $0.53 \pm 0.13, p=0.827$ ) (Table 2).

There was also no significant correlation between the psychophysical TDI score and the amplitude of OLF-N1 $(r=-0.20, p=$ $0.271)$ or OLF-P2 $(r=0.12, p=0.520)$.

Across-trial averaging in the time-frequency domain As shown in the CWT-AVERAGE transform (Figure 2), olfactory chemosensory stimulation elicited a small phase-locked increase in EEG signal power in the normosmic group. This phase-locked increase in EEG signal power appeared to be less 
Olfactory stimulation
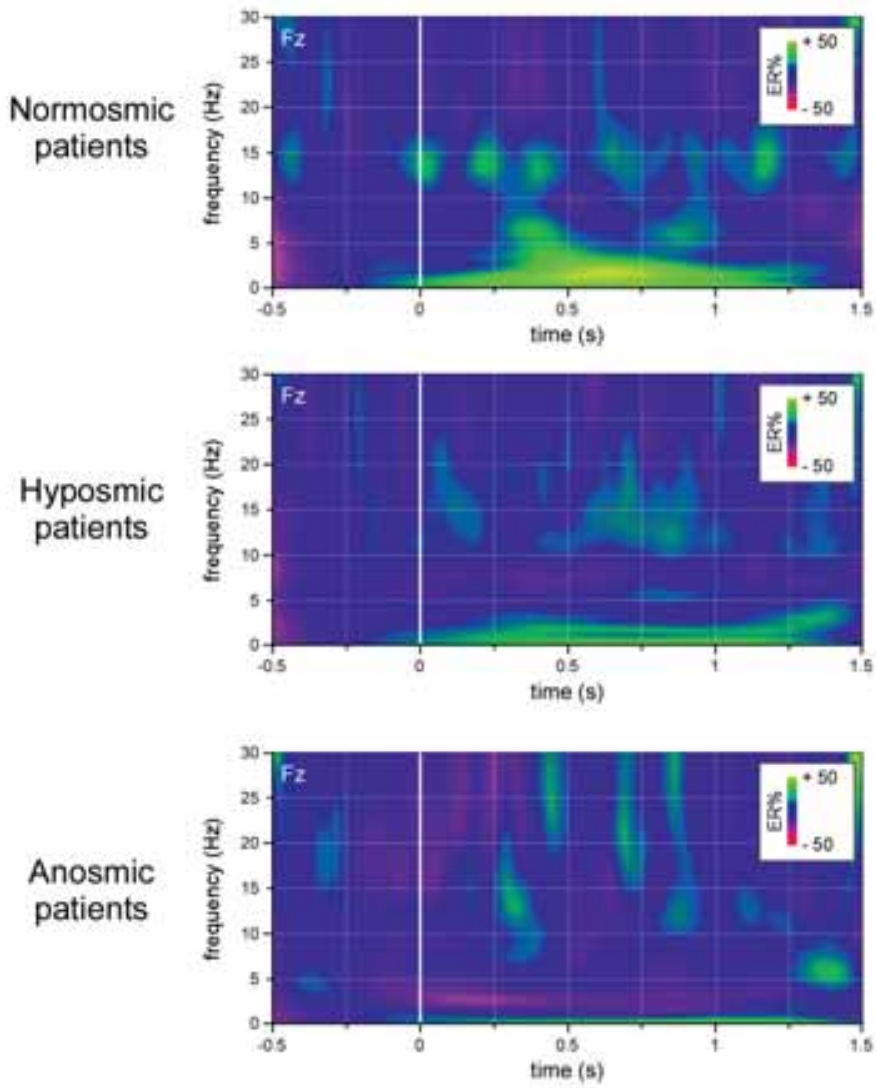

Trigeminal chemosensory stimulation
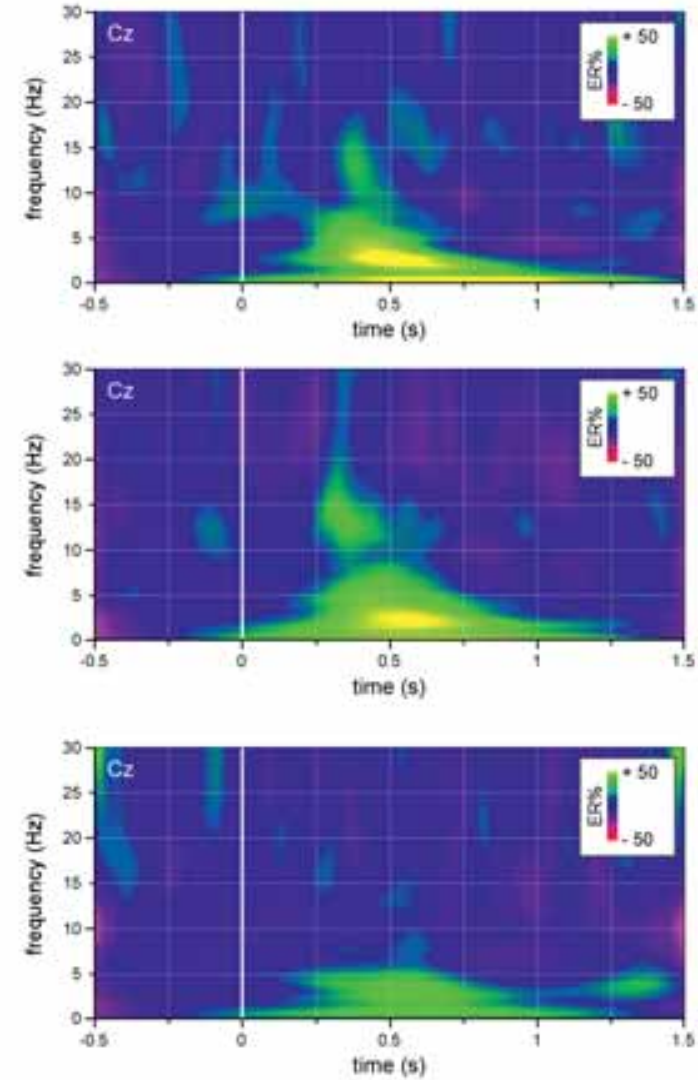

Figure 3. Time-frequency representation of the non-phase locked EEG responses to olfactory and trigeminal stimulation (CWT-SINGLE) in the normosmic, hyposmic and anosmic groups of patients.

Non phase-locked EEG responses were identified by performing across-trial averaging in the time-frequency domain, enhancing both phase-locked and non phase-locked EEG responses. Signal amplitude (group-level average; olfactory stimulation: electrode Fz vs. A1 A2; trigeminal stimulation: electrode $C z$ vs. A1A2) is expressed as a percentage increase or decrease relative to baseline ( -0.4 to $-0.1 \mathrm{~s})$ (ER\%). After olfactory stimulation, normosmic patients exhibit a clear and long-lasting increase of signal amplitude at low frequencies, referred to as OLF-TF1. In hyposmic patients, the magnitude of OLF-TF1 is reduced. In anosmic patients, this increase cannot be identified. Also note that trigeminal stimulation does not only elicit a phase-locked EEG response (TRI-TF1) but also induces a long-lasting desynchronisation of alpha-band EEG rhythms (8-12Hz) and a non phase-locked increase in EEG signal amplitude peaking approximately $350 \mathrm{~ms}$ after stimulus onset and centered around 10-15 Hz.

pronounced in the group of hyposmic patients and was hardly identifiable in the group of anosmic patients.

As show in Figure 3, the CWT-SINGLE transform revealed clearer EEG responses to olfactory stimulation. In the normosmic group, we observed a long-lasting increase in the amplitude of lowfrequency EEG oscillations. The magnitude of this response was decreased in the hyposmic group, and the response was not visible in the anosmic group. An estimate of the latency and frequency of the response obtained in the CWT-SINGLE transform was obtained by locating the peak amplitude within 300-1000 ms and 3-7 Hz (electrode Fz). An estimate of the amplitude of the response was obtained by considering the maximum amplitude value within this region of interest.

In the time domain, the EEG response within this ROI peaked at $643 \pm 197,557 \pm 207$ and $641 \pm 298 \mathrm{~ms}$ in the normosmic, hyposmic and anosmic groups, respectively. These differences were not significant $(F=0.469 ; p=0.630)$.

In the frequency domain, the EEG response within this ROI peaked at $4.4 \pm 1.5,4.6 \pm 1.5$ and $4.9 \pm 1.4 \mathrm{~Hz}$ in the normosmic, hyposmic and anosmic groups, respectively. These differences were not significant $(F=0.263 ; p=0.771)$.

The magnitude of the EEG response within this $\mathrm{ROI}$ was +64 
Normosmic vs Hyposmic/Anosmic

\section{Normosmic vs Hyposmic}

\section{Normosmic vs Anosmic}

\section{OLF-N1}
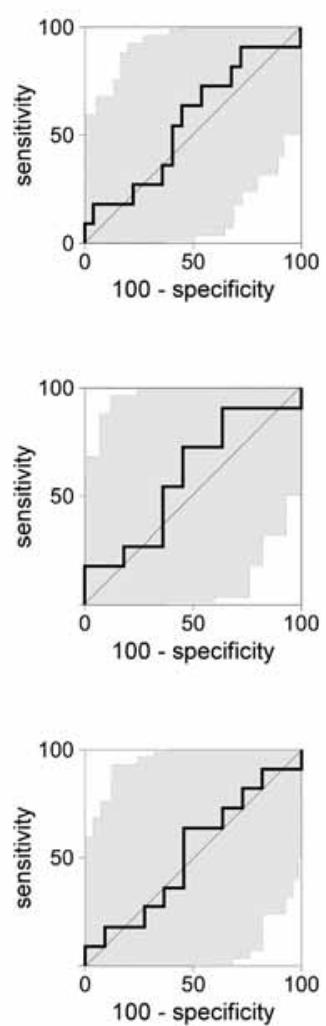

OLF-P2
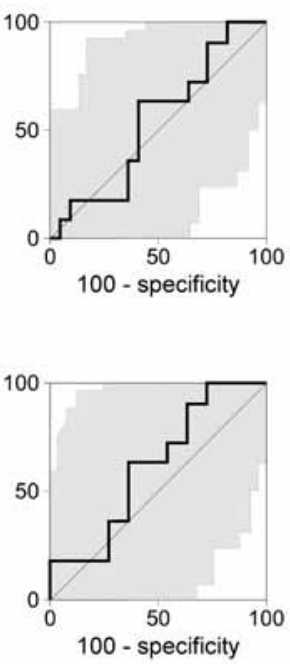

OLF-TF1

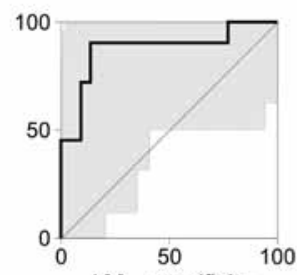

100 - specificity
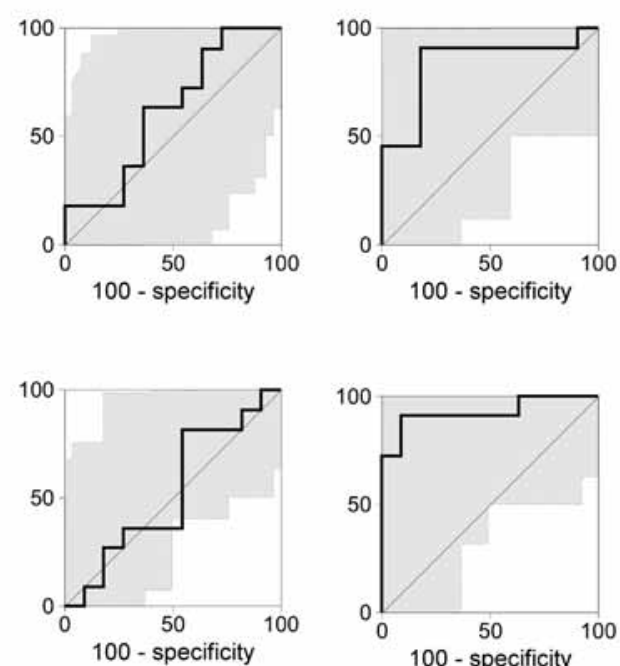

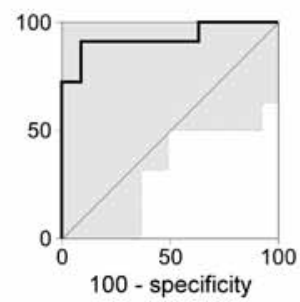

- ROC curve

$\square 95 \%$ confidence interval

Figure 4. Receiver Operating Characteristic (ROC) analysis. ROC curves were computed to estimate the discrimination performance of each of the different measures of the EEG response to olfactory stimulation identified using across-trial averaging in the time-domain (OLF-N1 and OLF-P2) and across-trial averaging in the time-frequency domain (OLF-TF1). The shaded areas represent the $95 \%$ confidence interval of the obtained curves.

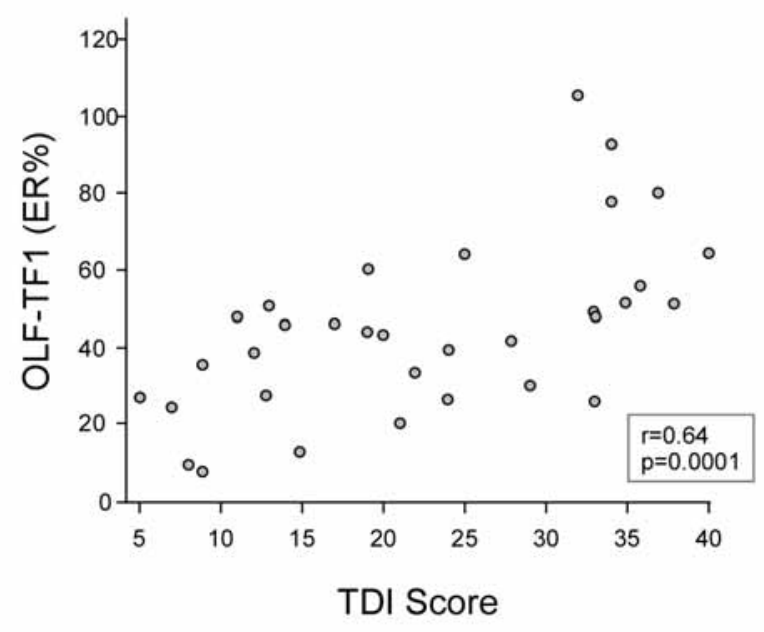

Figure 5. Correlation between psychophysical olfactory performance (TDI score) and the magnitude of the OLF-TF1 response to olfactory stimulation identified in the CWT-SINGLE transform. Note the significant positive correlation between the TDI score and OLF-TF1 magnitude ( $r=$ $0.64, p=0.0001)$.
Table 1. Latency and amplitude of EEG responses to olfactory and trigeminal stimulation obtained after time-domain averaging (measured at $\mathrm{Cz}$ ).

\begin{tabular}{|c|c|c|c|c|}
\hline LATENCY & OLF-N1 & OLF-P2 & TRI-N1 & TRI-P2 \\
\hline Normosmic & $357 \pm 32 \mathrm{~ms}$ & $\begin{array}{c}566 \pm \\
101 \mathrm{~ms}\end{array}$ & $398 \pm 37 \mathrm{~ms}$ & $533 \pm 32 \mathrm{~ms}$ \\
\hline Hyposmic & $398 \pm 42 \mathrm{~ms}$ & $\begin{array}{c}570 \pm \\
102 \mathrm{~ms}\end{array}$ & $405 \pm 29 \mathrm{~ms}$ & $579 \pm 75 \mathrm{~ms}$ \\
\hline Anosmic & $383 \pm 38 \mathrm{~ms}$ & $678 \pm 90 \mathrm{~ms}$ & $406 \pm 55 \mathrm{~ms}$ & $568 \pm 100 \mathrm{~ms}$ \\
\hline \multicolumn{5}{|l|}{ AMPLITUDE } \\
\hline Normosmic & $-3.4 \pm 2.2 \mu \mathrm{V}$ & $4.9 \pm 2.1 \mu \mathrm{V}$ & $-6.3 \pm 3.6 \mu \mathrm{V}$ & $7.3 \pm 3.4 \mu \mathrm{V}$ \\
\hline Hyposmic & $-2.7 \pm 2.0 \mu \mathrm{V}$ & $4.0 \pm 1.6 \mu \mathrm{V}$ & $-5.7 \pm 4.4 \mu \mathrm{V}$ & $8.4 \pm 3.9 \mu \mathrm{V}$ \\
\hline Anosmic & $-3.3 \pm 2.3 \mu \mathrm{V}$ & $5.2 \pm 2.2 \mu \mathrm{V}$ & $-4.3 \pm 3.5 \mu \mathrm{V}$ & $6.0 \pm 2.1 \mu \mathrm{V}$ \\
\hline
\end{tabular}


Table 2. Discrimination performance of the phase-locked and non phase-locked EEG responses to olfactory stimulation. (AUC: Area Under the Curve; p: p-value, Se: Sensitivity, Sp: Specificity).

\begin{tabular}{|c|c|c|c|c|c|c|c|c|c|c|c|c|}
\hline & \multicolumn{4}{|c|}{ Normosmic vs Hyposmic/Anosmic } & \multicolumn{4}{|c|}{ Normosmic vs Hyposmic } & \multicolumn{4}{|c|}{ Normosmic vs Anosmic } \\
\hline & AUC & $\mathrm{p}$ & $\mathrm{Se}$ & Sp & AUC & $p$ & Se & $\mathrm{Sp}$ & AUC & $p$ & Se & $\mathrm{Sp}$ \\
\hline OLF-N1 & $\begin{array}{c}0.56 \\
\pm 0.11\end{array}$ & 0.600 & $73 \%$ & $45 \%$ & $\begin{array}{c}0.59 \\
\pm 0.13\end{array}$ & 0.461 & $73 \%$ & $55 \%$ & $\begin{array}{c}0.52 \\
\pm 0.13\end{array}$ & 0.874 & $64 \%$ & $55 \%$ \\
\hline OLF-P2 & $\begin{array}{c}0.54 \\
\pm 0.11\end{array}$ & 0.668 & $64 \%$ & $59 \%$ & $\begin{array}{c}0.62 \\
\pm 0.13\end{array}$ & 0.344 & $100 \%$ & $27 \%$ & $\begin{array}{c}0.53 \\
\pm 0.13\end{array}$ & 0.827 & $82 \%$ & $45 \%$ \\
\hline OLF-TF1 & $\begin{array}{c}0.88 \\
\pm 0.07\end{array}$ & 0.0001 & $91 \%$ & $86 \%$ & $\begin{array}{l}0.84 \\
\pm 0.1\end{array}$ & 0.0006 & $91 \%$ & $82 \%$ & $\begin{array}{c}0.93 \\
\pm 0.06\end{array}$ & 0.0001 & $91 \%$ & $91 \%$ \\
\hline
\end{tabular}

$\pm 23,+41 \pm 13$ and $+30 \pm 15 \%$ in the normosmic, hyposmic and anosmic groups, respectively. These differences were significantly different $(F=10.525 ; p<0.001)$. Posthoc pairwise comparisons revealed that the magnitude of the EEG response was significantly different between normosmic and hyposmic patients $(p=0.015)$, as well as between normosmic and anosmic patients $(p<0.001)$.

The magnitude of this EEG response was able to discriminate 1) between normosmic and hyposmic/anosmic patients (AUC = $0.88 \pm 0.07, p=0.0001) ; 2$ ) between normosmic and hyposmic patients ( $A \cup C=0.84 \pm 0.10, p=0.0006$ ); and 3 ) between normosmic and anosmic patients ( $A U C=0.93 \pm 0.06, p=0.0001$ ). Using the cutoff value associated with the greatest Youden index, the measure of the EEG response identified in the CWTSINGLE transform had 1) a sensitivity of $91 \%$ and a specificity of $86 \%$ to discriminate between normosmic and hyposmic/ anosmic patients; 2 ) a sensitivity of $91 \%$ and a specificity of $82 \%$ to discriminate between normosmic and hyposmic patients and 3) a sensitivity of $91 \%$ and a specificity of $91 \%$ to discriminate between normosmic and anosmic patients (Figure 4, Table 2). The discrimination performance of the EEG response identified in the CWT-SINGLE transform was significantly greater than the discrimination performance of OLF-N1 and OLF-P2 identified in the time domain, when assessing the ability to discriminate between 1) normosmic and hyposmic/anosmic patients (CWTSINGLE vs. OLF-N1: $\mathrm{p}=0.0087$; CWT-SINGLE vs. OLF-P2: $\mathrm{p}=$ 0.0188 ); and 2) normosmic and anosmic patients (CWT-SINGLE vs. OLF-N1: $p=0.0032 ;$ CWT-SINGLE vs. OLF-P2: $p=0.0087$ ); but 3) not when assessing the ability to discriminate between normosmic and hyposmic patients (CWT-SINGLE vs. OLF-N1: $\mathrm{p}=$ 0.070, CWT-SINGLE vs. OLF-P2: $\mathrm{p}=0.165$ ).

Finally, there was a significant correlation between the TDI score and the magnitude of the EEG response identified in the CWTSINGLE transform $(r=0.64, p=0.0001)$ (Figure 5). This correlation was also present when considering separately the threshold $(T)$, discrimination (D) and identification (I) scores (T: $r=0.64, p=$ $0.0001, D: r=0.49, p=0.0036, \mathrm{l}: r=0.48, p=0.0049$ ).

Trigeminal stimulation

Across trial averaging in time-domain

As shown in the group-level average waveforms (Figure 1), trigeminal stimulation elicited, at electrode $C z$, a negative deflection (TRI-N1) followed by a positive deflection (TRI-P2) in the three groups of patients (Table 1).

The latency of TRI-N1 was $398 \pm 37,405 \pm 29$ and $406 \pm 55$ ms in the normosmic, hyposmic and anosmic groups, respectively. The latency of TRI-P2 was $533 \pm 32,579 \pm 75$ and $568 \pm 100 \mathrm{~ms}$ in the normosmic, hyposmic and anosmic groups, respectively.

The amplitude of TRI-N1 was $-6.3 \pm 3.6,-5.7 \pm 4.4$ and $-4.3 \pm 3.5$ $\mu \mathrm{V}$ in the normosmic, hyposmic and anosmic groups, respectively. The amplitude of TRI-P2 was 7.3 $\pm 3.4,8.4 \pm 3.9$ and $6.0 \pm$ $2.1 \mu \mathrm{V}$ in the normosmic, hyposmic and anosmic groups, respectively. These differences were not significant (TRI-N1: $F=0.588 ; p$ $=0.564 ;$ TRI-P2: $F=1.608 ; p=0.223$ ).

Neither the amplitude of TRI-N1 nor the amplitude of TRI-P2 were able to discriminate 1) between normosmic and hyposmic/ anosmic patients (TRI-N1: AUC $=0.62 \pm 0.10, p=0.233$; TRI$\mathrm{P} 2$ : $\mathrm{AUC}=0.51 \pm 0.12, \mathrm{p}=0.947) ; 2$ ) between normosmic and hyposmic patients (TRI-N1: AUC $=0.57 \pm 0.14, p=0.610$; TRI-P2: $A \cup C=0.59 \pm 0.13, p=0.507)$; and 3 ) between normosmic and anosmic patients (TRI-N1: AUC $=0.67 \pm 0.12, \mathrm{p}=0.164$, TRI-P2: AUC $=0.60 \pm 0.13, p=0.441$ ) (Table 2).

There was also no significant correlation between the psychophysical TDI score and the amplitude of TRI-N1 $(r=-0.197, p=$ $0.271)$ or TRI-P2 ( $r=0.116, p=0.520)$.

Across trial averaging in the time-frequency domain As shown in Figure 2, the CWT-AVERAGE transform revealed a phase-locked EEG response corresponding to the trigeminal CSERP in the three groups of patients. In addition to 1) this 
phase-locked EEG response, the CWT-SINGLE transform also showed 2) a long-lasting desynchronization of alpha-band (8$12 \mathrm{~Hz}$ ) oscillations starting approximately $600 \mathrm{~ms}$ after stimulus onset and 3) a non phase-locked increase in EEG signal amplitude peaking approximately $350 \mathrm{~ms}$ after stimulus onset and centred around $10-15 \mathrm{~Hz}$. These responses were similar to those reported in our previous study ${ }^{(18)}$. As described in the Methods section, an estimate of the latency and frequency of the response obtained in the CWT-SINGLE transform was obtained by locating the peak amplitude within $200-600 \mathrm{~ms}$ and $2-7.5 \mathrm{~Hz}$ (electrode $\mathrm{Cz}$ ). An estimate of the amplitude of the response was obtained by considering the maximal amplitude value within this region of interest.

In the time domain, the EEG response within this ROI peaked at $482 \pm 114,494 \pm 39$ and $486 \pm 126 \mathrm{~ms}$ in the normosmic, hyposmic and anosmic groups, respectively.

In the frequency domain, the EEG response within this ROI peaked at $4.5 \pm 1.9,3.9 \pm 1.4$ and $4.2 \pm 1.6 \mathrm{~Hz}$ in the normosmic, hyposmic and anosmic groups, respectively. These differences were not significant $(F=0.334 ; p=0.718)$.

The magnitude of the EEG response within this $\mathrm{ROI}$ was $+89 \pm$ $78,+83 \pm 44$ and $+62 \pm 33 \%$ in the normosmic, hyposmic and anosmic groups, respectively. These differences were not significant $(F=0.751 ; p=0.480$ ). There was no significant correlation between the magnitude of the EEG response and the TDI score $(r=0.12, p=0.506)$.

\section{Discussion}

In our previous study ${ }^{(18)}$, we showed that time-frequency analysis can be used to significantly enhance the signal-to-noise ratio of the EEG responses elicited by chemosensory stimulation in healthy subjects. Here, our aim was to demonstrate the clinical usefulness of this approach. For this purpose, we chose to retrospectively analyse EEG recordings obtained in a group of anosmic, hyposmic and normosmic patients examined in a routine clinical setting. Hence, as compared to our previous study, the EEG responses were obtained using a smaller number of trials ( 20 vs. 60 stimuli) and scalp electrodes (3 vs. 64 electrodes).

\section{EEG Responses to olfactory stimulation}

The discrimination performance of olfactory ERPs identified using conventional time-domain averaging was poor. In contrast, the discrimination performance of the EEG responses to olfactory stimulation identified in the time-frequency domain (OLF-TF1) was markedly higher ("normosmic" vs "hyposmic + anosmic": $p=0.0001$, Sensitivity: $91 \%$, Specificity: $86 \%$; "normosmic" vs "hyposmic": $p=0.0006$, Sensitivity: $91 \%$, Specificity: 82\%; "normosmic" vs "anosmic": $p=0.0001$, Sensitivity: $91 \%$, Specificity: 91\%) (Figure 4). The ability of OLF-TF1 to discriminate between "normosmic" and "hyposmic + anosmic" groups; and between "normosmic" and "anosmic" groups was significantly higher than the discrimination performance of OLF-N1 $(p=$ 0.009 and $p=0.019$, respectively) and OLF-P2 ( $p=0.003$ and $p=0.009$, respectively). In contrast, the ability of OLF-TF1 to discriminate between the "normosmic" and "hyposmic" group was not significantly higher as compared to OLF-N1 $(p=0.070)$ and OLF-P2 ( $p=0.165)$.

Of note, OLF-N1 and OLF-P2 peaks were clearly identified in one of the patients categorized as anosmic based on the psychophysical results, suggesting that the olfactory function of this patient was at least partially preserved.

The signal-to-noise ratio of the EEG responses to olfactory stimulation identified in the CWT-SINGLE transform was much greater than the signal-to-noise ratio of the EEG responses identified using conventional time-domain averaging (OLF-N1 and OLF-P2), as well as that of the EEG responses identified in the CWT-AVERAGE transform. Since the CWT-SINGLE transform highlights both EEG responses that are phase-locked and non phase-locked across trials, the greater signal-to-noise ratio and, consequently, the greater discrimination performance of the responses obtained using the CWT-SINGLE transform is most likely explained by the fact that the EEG responses elicited by olfactory stimulation are not strictly time-locked to the onset of the stimulus. The poor stationarity of the elicited responses could be explained by a significant amount of temporal jitter ${ }^{(18)}$

In patients suffering from an olfactory disorder, we could expect that the responses to olfactory stimuli are even more desynchronised across trials as compared to healthy control subjects, due to a pathological transduction, conduction and/or processing of the olfactory input at peripheral and/or central level. In chronic rhinosinusitis, inflammatory processes induce apoptotic pathological changes in the olfactory neuroepithelium and alter the mucus composition and hence the olfactory transduction process ${ }^{(33-37)}$. In patients suffering from post-traumatic or postinfectious olfactory loss, the olfactory neuroepithelium appears to be disorganized, with a reduced number of intact ciliated olfactory receptor neurons ${ }^{(38,39)}$. At central level, it is well known that the olfactory bulb of patients with post-traumatic or postinfectious olfactory loss show lesions and/or a reduced volume ${ }^{(40,41)}$. Finally, subcortical and cortical lesions can also contribute to olfactory dysfunction, for example, in post-traumatic olfactory loss, or olfactory loss associated with a neurodegenerative disease. All these pathological changes are likely to affect the transduction, conduction and generation of the olfactory signal and, hence, are likely to increase the temporal jitter of the EEG responses elicited by olfactory stimulation. Therefore, time-frequency analysis of EEG responses following olfactory stimulation could be particularly useful to characterise olfactory function in patients suffering from olfactory disorder. 
Here, within the present study conditions, we found that the CWT-SINGLE transform was more reliable than conventional time-domain averaging to evaluate objectively the olfactory function of patients. At the individual level, using the magnitude of the OLF-TF1 response associated with the highest Youden index, we were able to discriminate between normosmic and hyposmic/anosmic patients with a high sensitivity and specificity. Hence, time-frequency analysis of the EEG responses to olfactory stimulation could constitute a useful clinical diagnostic tool complementing the standard clinical workup of patients presenting with an olfactory disorder such as psychophysical testing and MRI. Nevertheless, it is important to highlight that the cut-off values discriminating between normosmic and hyposmic/anosmic patients obtained in the present study should not be considered as standard values as these could change as a function of the stimulation paradigm (e.g. inter-stimulus interval, type of odorant, stimulus intensity) as well as the EEG acquisition setup. Hence, clinicians aiming to implement this approach should first build their own reference values based on a control population. Furthermore, it should be stressed that our results are based on a relatively small sample of patients (33 patients). Further studies, reproducing our results in a larger group of patients are thus clearly needed to establish our approach in clinical routine.

In this study, we found a significant correlation between the orthonasal psychophysical score measured by the Sniffin' Sticks test and the magnitude of the OLF-TF1 response identified in the CWT-SINGLE transform. Such a correlation was absent for the OLF-N1 and OLF-P2 responses identified using conventional time-domain averaging, at least in the present sample under the present recording positions. Previous studies that attempted to correlate measures of the electrophysiological response to olfactory stimulation with TDI scores also showed no clear correlation with the amplitude of olfactory $\mathrm{N} 1$ and $\mathrm{P} 2$ peaks ${ }^{(42,43)}$. The fact that OLF-TF1 correlated with olfactory perception could be explained by the greater signal-to-noise ratio of this response as compared to the OLF-N1 and OLF-P2 responses. Hence, timefrequency analysis could be of particular interest for the clinical evaluation of patients, as it could provide an objective measure of olfactory function.

Finally, the contrast between the high discrimination performance of our measures obtained using time-frequency analysis and the low discrimination performance of our present measures obtained using conventional time-domain averaging should be tempered by the fact that, in the context of clinical evaluation, the presence of an OERP is not only determined by amplitude and/or latency criteria, but also integrates the visual interpretation of the shape of the ERP waveform, as well as its scalp topography. Here, it is important to highlight that, in the sample of the present study, no olfactory ERP were identified visually in 5 out of 11 normosmic patients, whereas time-frequency analysis miscategorised only 1 normosmic patient. This variability in the ERP response is also the reason why, so far in medico-legal cases, the presence of OERP is used as an indicator of the presence of olfactory function whereas the absence of OERP is not used as an indicator of missing olfactory function.

\section{EEG responses to trigeminal stimulation}

Neither the EEG responses to trigeminal stimulation obtained using conventional time-domain analysis (TRI-N1 and TRIP2), nor the trigeminal EEG responses obtained using timefrequency analysis (TRI-TF1) were able to discriminate between the three groups of patients. This contrasts with the results of previous studies showing that patients suffering from anosmia have significantly decreased EEG trigeminal responses as compared to normal subjects ${ }^{(44-46)}$. The lack of a significant difference between the trigeminal responses obtained in our group of normosmic, hyposmic and anosmic patients could be explained by the relatively small sample size, as well as by the heterogeneity of the conditions associated with olfactory dysfunction.

\section{Conclusion}

The present study shows that time-frequency analysis of the EEG responses to olfactory stimulation could be used as an effective diagnostic tool for the objective clinical evaluation of patients presenting with an olfactory disorder, as the technique is able to discriminate between normosmic patients and hyposmic/anosmic patients with a high sensitivity and specificity.

There is currently no effective treatment for olfactory dysfunction. Therefore, clinicians agree that it is essential to provide prognosis and appropriate counselling to patients suffering from an olfactory deficit. Several prognostic factors have been described in the literature, such as the results of psychophysical testing, age, sex, duration of the disease and olfactory bulb volume $^{(47-50)}$. Regarding OERPs, it has been shown that the presence of a measurable OERP is linked with a better outcome of patients with post-infectious olfactory loss ${ }^{(51)}$. Future studies should examine whether time-frequency analysis of the EEG responses to olfactory stimulation could provide additional information concerning the prognosis of recovery.

\section{Acknowledgement}

Dr. Huart is MD PhD student supported by the Fund for Scientific Research (F.R.S.-F.N.R.S.) of the French-Speaking Community of Belgium. Dr. Mouraux has received support from the Pain-Research EFIC-Grünenthal Grant 2008 (EGG), the IASP Early Career research Grant, and a from a Marie Curie European Reintegration Grant (ERG). Prof. Thomas Hummel received a grant from the Roland-Ernst-Stiftung. 


\section{Authorship contribution}

Conceived and designed the study: $\mathrm{CH}, \mathrm{PR}, \mathrm{TH}, \mathrm{AM}$. Analyzed the data: $\mathrm{CH}, \mathrm{AM}$. Wrote the paper: $\mathrm{CH}, \mathrm{PR}, \mathrm{TH}, \mathrm{AM}$.

\section{Conflict of interest}

The authors declare that no competing interests exist.

\section{References}

1. Gottfried JA. Smell: central nervous processing. Adv Otorhinolaryngol. 2006; 63: 44-69.

2. Landis BN, Konnerth CG, Hummel T. A study on the frequency of olfactory dysfunction. Laryngoscope. 2004; 114: 1764-1769.

3. Neuland C, Bitter T, Marschner H, Gudziol H, Guntinas-Lichius O. Health-related and specific olfaction-related quality of life in patients with chronic functional anosmia or severe hyposmia. Laryngoscope. 2011; 121: 867-872.

4. Hummel T, Kobal G, Gudziol H, Mackay-Sim A. Normative data for the "Sniffin' Sticks" including tests of odor identification, odor discrimination, and olfactory thresholds: an upgrade based on a group of more than 3,000 subjects. Eur Arch Otorhinolaryngol. 2007; 264: 237-243

5. Kobal G, Hummel T, Sekinger B, Barz S, Roscher S, Wolf S. "Sniffin' sticks": screening of olfactory performance. Rhinology. 1996; 34: 222-226.

6. Doty RL, Shaman P, Kimmelman CP, Dann MS. University of Pennsylvania Smell Identification Test: a rapid quantitative olfactory function test for the clinic. Laryngoscope. 1984; 94: 176-178.

7. Heilmann S, Strehle G, Rosenheim K, Damm M, Hummel T. Clinical assessment of retronasal olfactory function. Arch Otolaryngol Head Neck Surg. 2002; 128: 414-418.

8. Haehner A, Mayer AM, Landis BN, et al. High test-retest reliability of the extended version of the "Sniffin' Sticks" test. Chem Senses. 2009; 34: 705-711.

9. Rombaux P, Collet S, Martinage $S$, et al. Olfactory testing in clinical practice. B-ENT. 2009; 5 Suppl 13: 39-51.

10. Rombaux P, Mouraux A, Collet S, Eloy P, Bertrand B. Usefulness and feasibility of psychophysical and electrophysiological olfactory testing in the rhinology clinic. Rhinology. 2009; 47: 28-35.

11. Hummel T, Klimek L, Welge-Lussen A, al. Chemosensory evoked potentials for clinical diagnosis of olfactory disorders. HNO. 2000; 48: 481-485.

12. Rombaux P, Mouraux A, Bertrand B, Guerit JM, Hummel T. Assessment of olfactory and trigeminal function using chemosensory event-related potentials. Neurophysiol Clin. 2006; 36: 53-62

13. Pause BM, Krauel K. Chemosensory eventrelated potentials (CSERP) as a key to the psychology of odors. Int J Psychophysiol. 2000; 36: 105-122.

14. Hummel T, Barz S, Pauli E, Kobal G. Chemosensory event-related potentials change with age. Electroencephalogr Clin Neurophysiol. 1998; 108: 208-217.

15. Lotsch J, Hummel T. The clinical significance of electrophysiological measures of olfactory function. Behav Brain Res. 2006; 170: 78-83.

16. Boesveldt S, Haehner A, Berendse HW, Hummel T. Signal-to-noise ratio of chemosensory event-related potentials. Clin Neurophysiol. 2007; 118: 690-695.

17. Rombaux P, Bertrand B, Keller T, Mouraux A. Clinical significance of olfactory eventrelated potentials related to orthonasal and retronasal olfactory testing. Laryngoscope. 2007; 117: 1096-1101.

18. Huart C, Legrain V, Hummel T, Rombaux $P$, Mouraux A. Time-frequency analysis of chemosensory event-related potentials to characterize the cortical representaion of odors in humans. PLoS One 2012; 7(3): e33221. Doi:10.1371/journal.pone.0033221

19. Kobal G, Klimek L, Wolfensberger M, Gudziol $\mathrm{H}$, Temmel A, Owen CM, et al. Multicenter investigation of 1,036 subjects using a standardized method for the assessment of olfactory function combining tests of odor identification, odor discrimination, and olfactory thresholds. Eur Arch Otorhinolaryngol. 2000; 257: 205-211.

20. Kobal G, Hummel C. Cerebral chemosensory evoked potentials elicited by chemical stimulation of the human olfactory and respiratory nasal mucosa. Electroencephalogr Clin Neurophysiol. 1988; 71: 241-250.

21. Evans WJ, Cui L, Starr A. Olfactory eventrelated potentials in normal human subjects: effects of age and gender. Electroencephalogr Clin Neurophysiol. 1995; 95: 293-301.

22. Kobal G. Elektrophysiologische Untersuchungen des menschlichen Geruchsinns. Stuttgart: Georg Thieme; 1981.

23. Mouraux A, lannetti GD. Across-trial averaging of event-related EEG responses and beyond. Magn Reson Imaging. 2008; 26: 1041-1054.

24. Rombaux P, Mouraux A, Bertrand B, Guerit JM, Hummel T. Assessment of olfactory and trigeminal function using chemosensory event-related potentials. Neurophysiol Clin. 2006; 36: 53-62

25. Lorig TS, Matia DC, Peszka J, Bryant DN. The effects of active and passive stimulation on chemosensory event-related potentials. Int J Psychophysiol. 1996; 23: 199-205.

26. Hummel T, Kobal G. Olfactory event-related potentials. In: SA S, editor. Methods and new frontiers in neuroscience. Boca Raton: FL: CRC Press; 2002

27. Mouraux A, Guerit JM, Plaghki L. Nonphase locked electroencephalogram (EEG) responses to $\mathrm{CO} 2$ laser skin stimulations may reflect central interactions between $A$ partial partial differential- and C-fibre afferent volleys. Clin Neurophysiol. 2003; 114:
710-722.

28. Terwee $C B$, Bot SD, de Boer MR, van der Windt DA, Knol DL, Dekker J, et al. Quality criteria were proposed for measurement properties of health status questionnaires. J Clin Epidemiol. 2007; 60: 34-42.

29. Fischer JE, Bachmann LM, Jaeschke R. A readers' guide to the interpretation of diagnostic test properties: clinical example of sepsis. Intensive Care Med. 2003; 29: 10431051.

30. Bewick V, Cheek L, Ball J. Statistics review 13: receiver operating characteristic curves. Crit Care. 2004; 8: 508-512.

31. Youden W. Index for rating diagnostic tests Cancer. 1950; 3: 32-35.

32. DeLong ER, DeLong DM, Clarke-Pearson DL. Comparing the areas under two or more correlated receiver operating characteristic curves: a nonparametric approach. Biometrics. 1988; 44: 837-845.

33. Naessen R. An enquiry on the morphological characteristics and possible changes with age in the olfactory region of man. Acta Otolaryngol. 1971; 71: 49-62.

34. Hellings PW, Rombaux P. Medical therapy and smell dysfunction. B-ENT. 2009; 5 Suppl 13; 71-75.

35. Joshi H, Getchell ML, Zielinski B, Getchell TV. Spectrophotometric determination of cation concentrations in olfactory mucus. Neurosci Lett. 1987; 82: 321-326.

36. Getchell M, Mellert T. Olfactory mucus secretion. In: Getchell TV, Bartoshuk LM, Doty RL, Snow J (eds): Smell and Taste in health and disease, New York Raven Press, 1991, pp 83-95.

37. Downey $L L$, Jacobs JB, Lebowitz RA Anosmia and chronic sinus disease. Otolaryngol Head Neck Surg. 1996; 115: 24-28.

38. Moran DT, Jafek BW, Eller PM, Rowley JC. Ultrastructural histopathology of human olfactory dysfunction. Microsc Res Tech. 1992; 23: 103-110

39. Jafek BW, Murrow B, Michaels R, Restrepo D, Linschoten M. Biopsies of human olfactory epithelium. Chem senses. 2002; 27: 623-628.

40. Rombaux P, Mouraux A, Bertrand B, Nicolas $G$, Duprez T, Hummel T. Olfactory function and olfactory bulb volume in patients with post infectious olfactory loss Laryngoscope. 2006; 116: 436-439.

41. Rombaux P, Mouraux A, Bertrand B, Nicolas G, Duprez T, Hummel T. Retronasal and orthonasal olfactory function in relation to olfactory bulb volume in patients with posttraumatic loss of smell. Laryngoscope. 2006; 116: 901-905.

42. Welge-Luessen A, Kobal G, Wolfensberger M. Assessing olfactory function in laryngectomees using the Sniffin'Sticks test bat- 
tery and chemosensory evoked potentials. Laryngoscope. 2000; 110: 303-307.

43. Tateyama T, Hummel T, Roscher S, Post $H$, Kobal G. Relation of olfactory eventrelated potentials to changes in stimulus concentration. Electroencephalogr Clin Neurophysiol. 1998; 108: 449-455.

44. Frasnelli J, Schuster B, Hummel T. Interactions between olfaction and the trigeminal system: what can be learned from olfactory loss. Cereb Cortex. 2007; 17: 2268-2275.

45. Rombaux P, Mouraux A, Keller T, Hummel T. Trigeminal event-related potentials in patients with olfactory dysfunction. Rhinology. 2008; 46: 170-174.

46. Hummel T, Barz S, Lotsch J, Roscher S, Kettenmann B, Kobal G. Loss of olfactory function leads to a decrease of trigeminal sensitivity. Chem Senses. 1996; 21: 75-79.
47. Reden J, Mueller A, Mueller C et al. Recovery of olfactory function following closed head injury or infections of the upper respiratory tract. Arch Otolaryngol Head Neck Surg. 2006; 132: 265-269.

48. Hummel T, Lotsch J. Prognostic factors of olfactory dysfunction. Arch Otolaryngol Head Neck Surg. 2010; 136: 347-351.

49. London $B$, Nabet $B$, Fisher AR, White $B$, Sammel MD, Doty RL. Predictors of prognosis in patients with olfactory disturbance. Ann Neurol. 2008; 63: 159-166.

50. Rombaux P, Huart C, Deggouj N, Duprez T, Hummel T. Prognostic Value of Olfactory Bulb Volume Measurement for Recovery in Postinfectious and Posttraumatic Olfactory Loss. Otolaryngol Head Neck Surg. 2012; 147: 1136-1141.

51. Rombaux P, Huart C, Collet S, Eloy P, Negoias $S$, Hummel T. Presence of olfactory event- related potentials predicts recovery in patients with olfactory loss following upper respiratory tract infection. Laryngoscope 2010; 120: 2115-2118

\section{Dr Caroline Huart}

Institute of Neuroscience

Université catholique de Louvain

Brussels

Belgium

Tel: +32-2-764-9423

Fax: +32-2-764-5460

E-mail: caroline.huart@uclouvain.be

\section{ADVERTISEMENT}

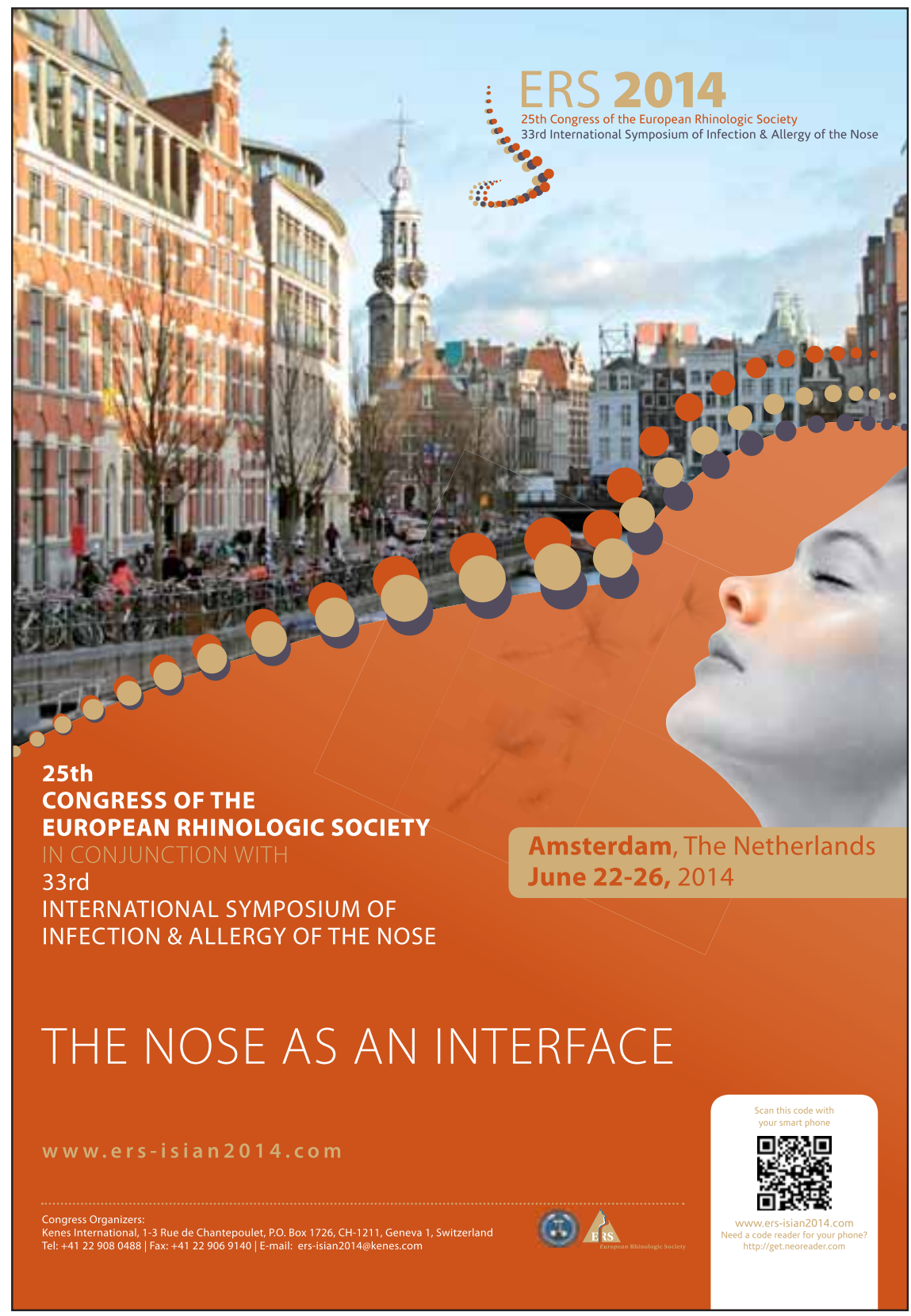

